A Profile of Public Health Educators in North Carolina's Local Health Departments.

By: Glascoff, Mary A., Johnson, Hans H., Glascoff, William J., Lovelace, Kay, Bibeau, Daniel L. PhD

Glascoff MA, Johnson H, Glascoff W, Lovelace K, \& Bibeau, DL (2005). A Profile of Public Health Educators in North Carolina's Local Health Departments. Journal of Public Health Management and Practice, 11(6): 528---537.

Made available courtesy of Lippincott, Williams \& Wilkins:

http://journals.Iww.com/iphmp/Abstract/2005/11000/A_Profile_of_Public_Health_Educato rs_in_North.9.aspx

***Reprinted with permission. No further reproduction is authorized without written permission from Lippincott, Williams \& Wilkins. This version of the document is not the version of record. Figures and tables are missing from this format of the document. $* * *$

Abstract:

The purpose of this study was to provide a profile of the public health education workforce in North Carolina. A survey was administered to all practicing health educators at local health departments (LHDs) in North Carolina. The study specifically attempted to answer four questions: (1) Who functions as health educators in LHDs in North Carolina? (2) What is the educational background and professional training of North Carolina LHD health educators? (3) What are the characteristics of health educators' positions in North Carolina? and (4) How do these characteristics of health educators (demographics and education) as well as their titles, job responsibilities, and supervisory relationships differ according to the size of the LHD? The study showed that most public health educators in North Carolina are white females; most do not have Certified Health Education Specialist certification; that younger health educators are more likely to have health education degrees; and that almost two thirds of public health educators have administrative responsibilities.

Keywords: public health education | public health educators | workforce enumeration | public health educator profiles | local health departments | North Carolina health departments

Article:

The public health workforce is the most essential part of the infrastructure needed to protect and promote, and ensure the public's health. 1 Understanding the composition of the workforce is critical for knowing if the requisite skills and competencies are available to public health agencies as they undertake this mission. To this end, public health workforce enumeration has occurred throughout the 20th and 21st centuries, starting with a 1920 study of full-time and parttime workers in municipal health departments by the American Public Health Association 
(APHA) and the US Public Health Service (USPHS). The USPHS has continued to enumerate the public health workforce with the last direct federal survey occurring in 1964. After that, estimates of the public health workforce were developed but direct surveys of the public health workforce were not conducted. 2

The call for enumerating and understanding the public health workforce has come from several key policy reports. An Institute of Medicine (IOM) report in 1988, The Future of Public Health, intensified interest in examining the public health workforce. 3 That report portrayed the public health system as in "disarray" and indicated that many, if not most, of public health workers did not have the appropriate professional public health training. The report called for developing a knowledge base about the public health workforce. The 1988 IOM report was followed in 2002 and 2003 with two studies that further examined the state of the public health system, The Future of the Public's Health in the 21st Century 4 and Who Will Keep the Public Healthy? 5 Fifteen years after the first IOM report on the public health system, major recommendations still focused on the having an adequate public health workforce and on increasing the qualifications and competencies of public health workers. An expert panel, convened at the Third Annual Public Health Workforce Development Meeting in early 2003, reviewed the work of the 2002 IOM committees. In that meeting, a strategic model for public health workforce development was discussed. The model had six elements; the first two elements were monitoring public health workforce composition and identifying competencies/developing curriculum. 6 Although there has not been a recent direct study of the US public health workforce, Gebbie et al 7 conducted a secondary analysis of data from existing workforce reports, surveys, and other studies to present a picture of the workforce at the national and local levels using occupational titles.

Approximately 34 percent of the public health workforce worked at the local level; 33 percent worked at the state level, 19 percent worked at the federal level, and 14 percent worked in other settings. Gebbie et al reported that of the 448,254 enumerated persons in professional occupational categories, public health nurses made up the largest segment (49,232 or 10.9\%). Health educators made up approximately 1 percent of the public health professional workforce with 2,230 persons enumerated.

Several researchers have studied the public health workforce in specific states. For example, to obtain an estimate of the public health workforce in Texas, Kennedy et al 8 sampled employers and potential employers in five categories. These included official state agencies with primary responsibility for providing one or more essential public health services, other state and regional agencies (eg, agriculture, human services, and public safety), local health departments (LHDs), other community-based agencies, and private, not-for-profit, or voluntary agencies. In the second stage of the study, individual public health workers were surveyed about themselves and their jobs. Of the estimated 7,731 public health professionals working in population-based public health services, 1.8 percent were estimated to be health educators. Although one half reported having a bachelor's degree and one fourth had a master's degree, only 7 percent reported having a 
major field of study in public health or a closely associated degree (eg, environmental health or health education). Richardson et al 9 surveyed 100 percent of the local public health agency administrators in Idaho and Wyoming regarding the staffing patterns and training of the professional and support staff in their agencies. LHDs differed in average size (101 mean full time equivalents [FTEs]/LHD in Idaho; 9.1 mean FTEs/LHD in Wyoming). One hundred percent of the LHDs in Idaho employed a health educator while only 4 percent of the LHDs in Wyoming employed a health educator. Fifty-one percent of Idaho LHD staff had previous public health experience or formal public health training; only 40 percent of staff in Wyoming had such training.

To understand the specific needs of rural communities, Hajat et al 10 compared the local public health agency workforce in rural, suburban, and metropolitan communities in a nationwide sample of 694 local public health agencies. The median number of health educators in each type of agency (rural, suburban, and metropolitan) was one. Survey respondents (public health administrators) in rural and metropolitan public health agencies reported that their greatest staffing needs were for public health nurses, environmental scientists, and health educators (in that order). Suburban respondents ranked health educators as their fourth greatest staffing need.

Mail 11 used data based on those with Certified Health Education Specialist (CHES) certification in the years 1981-1991 to develop a national profile of the health education workforce. Mail reported that of the total CHES individuals $(N=3,192)$ over those years, 30 percent held bachelor's degrees in health education, 12 percent in physical education, 10 percent in nursing, and 48 percent in other fields. Of the health educators reporting master's degrees, 56 percent held them in health education, 11 percent in public health, 5 percent in education, and 28 percent in other disciplines. Of the health educators renewing their CHES certification in 1991, there were 4 percent with less than 1-year experience, 10 percent with 1 to 6 years experience, 35 percent with 7 to 12 years, 28 percent with 13 to 18 years, 15 percent with 19 to 24 years, and 6 percent with 25 or more years experience. Thirty-one percent of individual renewing CHES certification worked in the public health or community health setting.

Two studies have looked specifically at health educators in the public health workforce. Clark et al 12 conducted a national random survey of LHD administrators to describe the health educator workforce. On the basis of a sample of 279 questionnaires and a weighting formula and interpolation to make projections, they reported a workforce that was approximately 80 percent female, 80 percent white, and 14 percent CHES certified. They further reported that 50 percent of the health educators held a BS/BA degree, 32 percent held an MPH degree, 12 percent held an 
MS degree, and 7 percent held a BN degree. Clark et al projected that there were between 3,570 and 4,040 public health education positions nationwide.

Finocchio et al 13 conducted a labor market study of public health educators in the San Francisco Bay Area. Their study focused on the number of MPH health educators on staff, the employer's hiring goals over the next 4-year period, and the importance of selected competencies. The respondents ( $\mathrm{N}=91$ employers) hired $210 \mathrm{MPH}$ health educators between 1995 and 1999. The health educators were employed in community-based organizations (about one half of all hires), county health departments (about one third of all hires), and national health associations, health maintenance organizations, and educational institutions (less than $10 \%$ of hires each). Importantly, while 69 percent of employers interviewed said that finding staff with bilingual competency was very important or important, 64 percent said that finding bilingual staff was very or fairly difficult. Similarly, 80 percent reported that finding staff with an ethnic background reflecting the clients served was very important or important and 62 percent reported that finding such staff was very or fairly difficult.

The purpose of the current study was to build on previous efforts at workforce enumeration by providing a profile of the public health education workforce in North Carolina. The study specifically attempted to answer four questions: (1) Who functions as health educators in LHDs in North Carolina? (2) What is the educational background and professional training of North Carolina LHD health educators? (3) What are the characteristics of health educators' positions in North Carolina? and (4) How do these characteristics of health educators (demographics and education) as well as their titles, job responsibilities, and supervisory relationships differ according to the size of the LHD? The research reported here is part of a larger study of the public health educator workforce in North Carolina, which was partially funded by the North Carolina Chapter of the Society of Public Health Educators (NC SOPHE). 14

\section{Public Health Education in North Carolina}

The governmental public health system in North Carolina employs public health educators at the state and local levels. At the state level, health education consultation and technical assistance services are provided via the Office of Healthy Carolinians/Health Education in the Division of Public Health of North Carolina's Department of Health and Human Services. At the local level, public health educators are employed in 79 county health departments and seven multicounty 
health departments. 15 The results of a recent survey 16 indicate that public health educators make up 3.6 percent of the total public health workforce in LHDs in North Carolina.

At the local level, there are five classifications of health education positions; from lowest to highest they are as follows: public health educator I, public health education specialist, public health educator II, public health educator supervisor, and public health educator III. The specifications for a public health educator I position stipulate a 4-year degree with a major in health education, although an individual may be hired as a health education trainee with a 4-year degree and work toward a public health educator I position. Beginning with the public health educator II position, the specifications stipulate a master's degree in public health education or in public health with a major in public health education. For the purposes of this study, the investigators considered a degree in health education to include degrees with majors in health education, community health education, health promotion, school health, worksite health promotion, and public health education. The specifications for health educator positions do not stipulate CHES certification.

Methods

Procedure

We mailed questionnaires to all health educators employed in North Carolina's 86 locally controlled health departments using a list that is routinely updated by the North Carolina Division of Public Health. Using mail survey method of Dillman, 17 we informed respondents that their responses would remain confidential; however, we used an identifying number to track nonrespondents. We followed up over a 2-month period, first by remailing the questionnaire, then by e-mail contact that included a copy of the questionnaire, and finally by telephone request. In the questionnaires, we asked about health educators' position titles, academic degrees, years of experience, CHES certification, and their demographics. In addition, we asked about their job responsibilities, whether or not they supervised others, the position title of their supervisor, the number of health educators in the agency, and the total FTEs employed by the agency.

First, we reviewed the literature on workforce enumeration and on public health infrastructure while we concurrently held conversations with the state director of health education, regional health education consultants, members of NC SOPHE, and other practicing health educators to develop a list of issues relevant to the public health education workforce and its development in 
North Carolina. Second, we developed a draft questionnaire. Third, the collaborating partners reviewed the questions for clarity. Fourth, we pilot tested the revised questionnaire with graduate health education students and four practicing North Carolina local health educators in one LHD. The health educators who completed the questionnaire critiqued it for clarity and content and gave us an estimate of the time needed to complete it. Fifth, on the basis of the respondents' feedback, we revised the questionnaire and submitted the study proposal including the questionnaire to the Institutional Review Boards at East Carolina University and the University of North Carolina at Greensboro. Both boards approved the study. Although four of the individuals participating in the pilot test also completed the final questionnaire, the study is descriptive and this should not be considered a threat to the validity of the data. Next, we describe the specific measures.

Measures included in the questionnaire were classified as either individual or organizational in nature. These two categories of measures allowed for independent analysis of both the health educators and the environment in which they worked.

Individual measures

These variables fell into two groups: (1) personal data and (2) training and experience. Questions used to gather personal data requested the gender, age, and race/ethnicity of respondents. Questions regarding the training and experience of respondents asked (1) if they were a CHES, (2) their degree majors, (3) the level of their degree/s (bachelor's, master's, and doctorate), (4) the name of their university, and (5) how long they had been employed as a health educator. In regard to their degrees, health educators were considered to have formal training in health education if they reported their major to be health education, community health education, health promotion, school health, worksite health promotion, or public health education.

\section{Organizational measures}

Organizational measures dealt with how health education was structured and administrated in an LHD, how many health educators were employed by the agency, and the overall size of the agency. Questions regarding the structure and administration of health education programs and the supervision of staff asked (1) the position title of the health educator being surveyed, (2) whether they administered a program, (3) whether they supervised other staff, and (4) if they were supervised by either a health educator or a staff member from another part of the health department. Although the above questions asked about the experience of the health educator who 
responded, we considered them organizational measures because they addressed how health education was structured and, further, the job position title and the supervisory relationships have implications for how public health education is practiced. Finally, we asked about the number of health educators employed in the agency and the total number of FTEs. We categorized local public health agencies employing 100 and less FTEs as small; health agencies employing 101 to 250 FTEs as medium; and health agencies employing 251 or more FTEs as large.

\section{Analysis}

Consistent with past public health and public health educator workforce studies, we used descriptive statistics to examine characteristics of the health educators, their education, certification, and experience. We also used descriptive statistics to describe whether health educators supervised programs, who supervised the health educators, the number of health educators in the agency, and the total FTEs. We used one-way analysis of variance (ANOVA) to examine formal health education training, health educator characteristics, and the structure and supervision of health education practice in North Carolina.

\section{Results}

This study resulted in a profile of practicing health educators employed in LHDs in North Carolina in the spring and summer of 2001. In all, 297 health educators employed in the 86 local public health agencies in North Carolina received the questionnaire; 205 health educators returned completed questionnaires for a response rate of 69.0 percent. Eighty-three of the 86 local public health agencies were represented in the final sample. At the time of the survey, two of the 86 local public health agencies did not employee a health educator and one agency's sole health educator did not respond. The results of the survey will be presented in table format, with the ramifications of those results presented in the discussion section that follows. Table 1 shows the agency size and number of health educators responding.

\section{Demographic description of the sample}

The first question we addressed was, "Who functions as health educators in LHDs in North Carolina?” Table 2 shows the demographic and educational characteristics of the respondents and the characteristics of the health educators' positions.

Education and employment 
The second question we addressed was, "What is the educational background and professional training of North Carolina's LHD health educators?” Table 3 presents information pertaining to that question. It should be noted that we asked about formal health education training. This training may differ from formal public health training. For instance, some of the sample may have graduated from a program within a school of public while others may have come from a health education program within a school of health and human performance. Table 3 also presents information about the number of health educators who were CHESs. Further analysis showed that of the CHES individuals, 88 percent $(n=22)$ had formal health education training (eight at the undergraduate level only, nine at the graduate level only, and five at both the undergraduate and graduate levels).

About 90 percent $(n=69)$ of the respondents who were younger than 30 years had formal health education training. For those 30 years and older, 80 (65\%) had formal health education training. A one-way ANOVA test with Bonferroni analysis showed a significant difference between the youngest and oldest health educators in terms of their formal training $(\mathrm{P}<=.05)$. Health educators younger than 30 were significantly more likely to have formal health education training than health educators older than 50 (Table 4).

Position titles, job responsibilities, and supervisory relationships

The third question we addressed was, "What are the characteristics of health educators' positions in North Carolina?” Table 5 also shows these characteristics. Most respondents used North Carolina Office of State Personnel Job Classification titles, as previously described.

Differences in health educator characteristics and positions by size of LHD and the size of the health education staff

The fourth question we addressed was, "How do the above characteristics of health educators (demographics and education) as well as their titles, job responsibilities, and supervisory relationships differ according to the size of the LHD?” Using a one-way ANOVA test with a Scheffe post hoc analysis, there was a significant difference in supervisory relationships. The largest LHDs, having 251 or more employees, were more likely to have a health educator supervise their own health educators than a smaller department. This relationship was significant $(\mathrm{P}<.01)$ when comparing the largest LHDs to both the medium and small LHDs. In addition, 
the smallest LHDs were significantly more likely to employ a lone health educator than a medium or large LHD ( $<$.01). Larger LHDs are more likely to employ five or more health educators than a medium LHD. There were no further significant differences in age, education, and job title and description by size of agency (Table 6).

\section{Discussion}

The purpose of the study was to provide a profile of the public health education workforce in North Carolina LHDs. In doing so, we answered four questions described in the results section. Taken together, the findings suggest the following implications.

First, the study showed that the public health education workforce does not adequately represent the population of the state. We found that health educators in North Carolina LHDs are primarily white females with mean age of 35 and with a bachelor's degree in health education. These health educators are usually not CHES certified. In contrast, the year 2000 US Census 18 found that 70.2 percent of the North Carolina population was white (not of Hispanic/Latino origin), 21.6 percent of the population was African American, and 4.7 percent of the population was Hispanic or Latino. These statistics closely mirror the racial distribution of whites and African Americans in the study sample; however, there was only one Hispanic health educator. North Carolina experienced a 393.9 percent growth of the Hispanic population from 1990 to 2000. In response, North Carolina LHDs increased the number of language interpreters employed by 129.4 percent between 1999 and 2000. 19 Thus, the underrepresentation of Hispanic public health educators presents concerns for workforce preparedness. We recommend that North Carolina universities preparing professional health educators (1) place an emphasis on recruiting Hispanic students into their programs and (2) consider requiring, or certainly encourage, Spanish language competency as part of the degree expectation.

Second, the public health education workforce studied here is overwhelmingly female (89\%). If male health educators are more appropriate for working in the areas of male sexuality and parenting, then the underrepresentation of males may result in a workforce preparedness issue. Why do fewer males work in health education positions? Our own experience in public health education professional preparation programs suggests that it is a pipeline issue. To understand this dynamic more fully, we have a few research questions. First, why do few males choose health education as a profession? What influences their perception of the profession? Do their perceptions influence their interest in the profession? Do their perceptions map reality? What is it that attracts males who do work as health educators in local public health agencies to those positions? We recommend that data be collected on their interests and motivations for working at 
the local level. Together, the information from the recommended research could be used to develop strategies for recruiting males into the profession. Further, salary studies could be used to compare health educators' salaries with those having commensurate skill and responsibilities. Data from such salary studies could be used to influence decision makers and possibly increase the salaries of local health educators. We anticipate that this would have payoffs in attracting all well-qualified professionals to local public health agencies.

Third, the health education workforce in North Carolina lacks CHES credentialing. Although the majority of the public health education workforce (75\%) has formal academic degrees in the field, only 12.2 percent of the respondents were CHES. This finding suggests that health departments may see the value of formal health education professional preparation, but are not seeking or requiring the CHES credential in the hiring process. This has important implications for health education training programs, for the profession and its professional organizations, and for individuals with CHES certification. To understand why employers are not hiring CHEScredentialed applicants, we need to know the extent to which it is a supply issue (insufficient applicants with these credentials) or a demand issue (the value-added of CHES credentialing is not seen by employers). If it is the latter, it is important to determine, in well-designed research studies, whether having CHES credentialing adds to the competence and the performance of health education professionals. Such studies are critical as national workforce preparedness efforts focus on certification and credentialing of the public health workforce.

Fourth, the finding that younger health educators are more likely to have health education degrees lends support to the idea that administrators value those with professional preparation in health education. It is possible that they recognize that trained health educators do a better job; it is possible that they are simply following the state criteria for health education positions. It is possible that the supply of health educators is currently sufficient for the demand in North Carolina. We would anticipate that this might be the case since there are five professional preparation programs in the state.

Fifth, the finding that almost two thirds of the respondents have some or primarily administrative responsibilities suggests that health educators are often viewed as having the skills and ability to take on administrative tasks. Of the 10 responsibilities for graduate-level health educators, administration is one. 20 This could have both negative and positive implications for the delivery of health education services. On one hand, if we want health educators to be supervised by other health educators, some will need to have some administrative responsibilities. Also, public health educators may be among the few health department employees who have formal public health educational backgrounds and thus an adequate understanding of assessment, assurance, and 
policy development. As such, having such a person in an administrative role could bring an advocate for population-focused prevention efforts to LHDs. Further, a health educator in an administrative role might have the organizational clout to get additional health education positions. On the other hand, a lone health educator with administrative responsibilities may not have adequate time to deliver population-focused prevention efforts.

Sixth, the situation of the lone health educator and the health educator supervised by non-health educators deserves special mention. Considering smaller LHDs were more likely to have lone health educators, it made sense that fewer were supervised by health educators. These professionals need to be able to clearly articulate and interpret their roles to others so that their skills will be appropriately used. Those supervised by clinical personnel may have a particularly difficult task if their supervisor sees their role as providing support to the clinical functions of the LHD. From the standpoint of professional preparation programs, this means that we need to prepare our graduates to be able to articulate their skills and training. Also, they need competencies in changing organizations, in advocating for appropriate population-based prevention efforts, and in using their skills in assessment, assurance, and policy development.

Consistent with national recommendations, this study reports an initial effort at understanding the public health education workforce in North Carolina. We are left with dilemmas and more research questions. Assuming, as did the IOM report, that the professional health education workforce should mirror the population, we need to know how to influence the recruitment, hiring, and retention of qualified Hispanic and male health educators. Many workforce preparedness efforts are promoting credentialing and certification of the public health workforce. An understanding of the value-added of CHES credentialing could add weight to the importance of credentialing and certification. Finally, much research is needed to understand the terrain of health educators in LHDs. With few colleagues who are also professionally prepared in health education or in public health, health educators may need well-developed support systems outside of their agency as well as a clearly articulated vision of how they can promote the public health enterprise.

\section{REFERENCES}

1. Woltring CS, Novick LF. Public health workforce: infrastructure's keystone. J Public Health Manag Pract. 2003;9:438-439.

2. Merrill J, Btoush R, Gupta M, Gebbie K. A history of public health workforce enumeration. J Public Health Manag Pract. 2003;9(6):459-470. 
3. Institute of Medicine. The Future of Public Health. Committee for the Study of the Future of Public Health, Division of Health Care Service. Washington, DC: National Academy Press; 1988.

4. Institute of Medicine. The Future of the Public's Health in the 21st Century. Committee on Assuring the Health of the Public in the 21st Century. Washington, DC: National Academy Press; 2002.

5. Institute of Medicine. Who Will Keep the Public Healthy: Educating Public Health Professionals for the 21st Century? Washington, DC: The National Academies Press; 2003.

6. Lichtveld MY, Cioffi JP. Public health workforce development: progress, challenges, and opportunities. J Public Health Manag Pract. 2003;9:43-50.

7. Gebbie K, Merrill J, Hwang I, Gebbie EN, Gupta M. The public health workforce in the year 2000. J Public Health Manag Pract. 2003;9(1):79-86.

8. Kennedy VC, Spears WD, Hardy DL, Moore FI. Public health workforce information: a statelevel study. J Public Health Manag Pract. 1999;5(3):10-19.

9. Richardson M, Casey S, Rosenblatt RA. Local health districts and the public health workforce: a case study of Wyoming and Idaho. J Public Health Manag Pract. 2001;7(1):37-48.

10. Hajat A, Stewart K, Hayes KL. The local public health workforce in rural communities. J Public Health Manag Pract. 2003;9(6):481-488.

11. Mail PD. A National profile of health educators: preliminary data from the first cohorts of CHES. J Health Educ. 1993;24(5):269-277.

12. Clark JK, Wood ML, Parrillo AV. Where public health educators are working: a national study of the hiring practices and manpower needs of local health departments. J Health Educ. 1998;29(4):250-257.

13. Finocchio LJ, Love MB, Sanchez EV. Illuminating the MPH health educator workforce: results and implications of an employer survey. Health Educ Behav. 2003;30(6):683-694.

14. Glascoff MA, Johnson HH, Lovelace K, Bibeau DL. Assessment of public health educator practice: health educator responsibilities. Health Promot Pract. 2005;6:89-96.

15. North Carolina Association of Local Health Directors. Available at: http://www.ncalhd.org/county.htm. Accessed August 17, 2004.

16. North Carolina State Center for Health Statistics. Local Health Department Staffing and Service Summary Fiscal Year 2003. Raleigh, NC: North Carolina Department of Health and Human Services, Division of Public Health; 2004. 
17. Dillman DA. Mail and Telephone Surveys: The Total Design Method. New York: WileyIntersciences; 1978.

18. US Census Bureau. State and county quickfacts: North Carolina quickfacts (2000). US Census Bureau. Available at: http://quickfacts.census.gov/qfd/states/37000.html. Accessed August 17, 2004.

19. North Carolina State Center for Health Statistics. Local Health Department Staffing and Service Summary Fiscal Year 2003. Raleigh, NC: North Carolina Department of Health and Human Services, Division of Public Health; 2004.

20. American Association for Health Education/Society for Public Health Education. Standards for the Preparation of Graduate-Level Health Educators. Washington, DC: American Association for Health Education/Society for Public Health Education; 1997. 